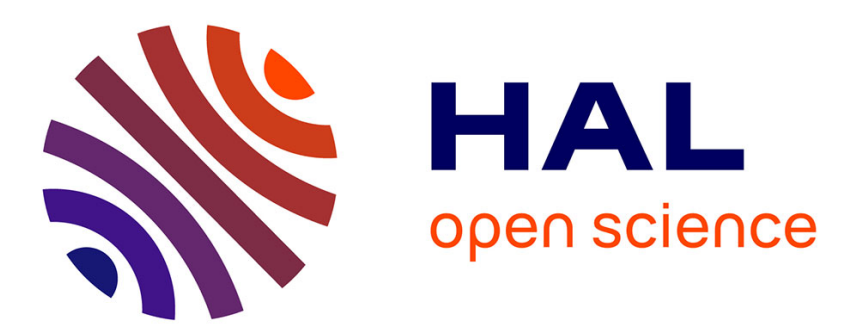

\title{
A new analysis of the cognitive radio jump-stay algorithm under the asymmetric model
}

Michel Barbeau, Gimer Cervera, Joaquin Garcia-Alfaro, Evangelos Kranakis

\section{To cite this version:}

Michel Barbeau, Gimer Cervera, Joaquin Garcia-Alfaro, Evangelos Kranakis. A new analysis of the cognitive radio jump-stay algorithm under the asymmetric model. ICC 2014 : IEEE International Conference on Communications, Jun 2014, Sidney, Australia. pp.1590 - 1595, 10.1109/ICC.2014.6883549 . hal-01264809

\section{HAL Id: hal-01264809 \\ https://hal.science/hal-01264809}

Submitted on 29 Jan 2016

HAL is a multi-disciplinary open access archive for the deposit and dissemination of scientific research documents, whether they are published or not. The documents may come from teaching and research institutions in France or abroad, or from public or private research centers.
L'archive ouverte pluridisciplinaire HAL, est destinée au dépôt et à la diffusion de documents scientifiques de niveau recherche, publiés ou non, émanant des établissements d'enseignement et de recherche français ou étrangers, des laboratoires publics ou privés. 


\title{
A New Analysis of the Cognitive Radio Jump-stay Algorithm Under the Asymmetric Model
}

\author{
Michel Barbeau*, Gimer Cervera ${ }^{\dagger}$, Joaquin Garcia-Alfaro $^{\ddagger}$ and Evangelos Kranakis* \\ * School of Computer Science, Carleton University, K1S 5B6, Ottawa, Ontario, Canada \\ Email: \{barbeau,kranakis\}@scs.carleton.ca \\ $\dagger$ Universidad Tecnológica Metropolitana, 97279, Merida, Yuc., Mexico \\ Email: gimer.cervera@utmetropolitana.edu.mx \\ $\ddagger$ Telecom SudParis, CNRS Samovar UMR 5157, Evry, France \\ Email: joaquin.garcia-alfaro@acm.org
}

\begin{abstract}
Underuse of the regulated radio spectrum is being addressed using a cognitive radio network approach termed dynamic spectrum access. Primary users have priority over the regulated radio spectrum. Secondary users may use the residual air time. We focus on the problem of meeting on a common channel by a group of secondary users. Under the asymmetric model, the secondary users have different sets of available channels. If the sets are not disjoint, they can eventually make rendezvous. The goal is to make the secondary users rendezvous on a common channel in a minimum amount of time. The jumpstay rendezvous algorithm has been created by Lin et al. to solve this problem. We develop a new analysis for the two-user expected time to rendezvous in the jump-stay rendezvous algorithm, under the asymmetric model, that better reflects its performance.
\end{abstract}

Keywords-Channel selection, cognitive radio network, cognitive wireless network, dynamic spectrum access, jump-stay algorithm, rendezvous.

\section{INTRODUCTION}

While the non regulated radio spectrum is too crowded, the regulated radio spectrum is not fully used. It has more potential than is currently being utilized. This issue is being addressed using a cognitive radio network approach named dynamic spectrum access. There are primary users, having priority over the regulated radio spectrum. Secondary users may use the residual air time. They can communicate over idle channels of the regulated radio spectrum as long as they do not create interference to the primary users. Meeting and establishing a multipoint network on a common channel by a group of secondary users is a challenging and intricate question.

We address the problem of selecting a common communication channel, among $m$ possible channels, between secondary users in a cognitive radio network, where $m$ is a positive integer. The issue has been addressed either by using a central controller, a distributed approach with dedicated common control channel or a distributed blind rendezvous approach. In this paper, the focus is on the distributed blind rendezvous approach. Each participant hops over a set of channels attempting to make rendezvous with other secondary users. Secondary users may have a common channel set (the symmetric model) or different, but non disjoint channel sets (the asymmetric model).
There are two conditions for a successful rendezvous: a successful protocol handshake and being on the same channel during a time slot. These two aspects can be considered separately. Probability-wise, they can be modelled individually and independently. The probability of a successful rendezvous is the product of the probability of a successful protocol handshake and probability of being on the same channel during a time slot. In this paper, the focus is on the latter aspect.

Lin et al. have introduced the jump-stay rendezvous algorithm that establishes rendezvous on a common channel between an arbitrary number of secondary users [1]-[3]. Rendezvous is achieved when all users meet on a common channel, if such a channel exists. The goal is to make the users rendezvous on a common channel in a minimum number of time slots. The algorithm of Lin et al. makes abstraction of any handshake communication protocol actually needed to make rendezvous.

We revisit the work of Lin et al. Since it does better than the previous version, we solely refer to the enhanced jump-stay rendezvous algorithm [1]. For the sake of simplicity, we omit the enhanced qualifier and refer to it as the jumpstay rendezvous algorithm. While the original logic remains the same, we develop a new analysis for the expected time to rendezvous (TTR) in the jump-stay rendezvous algorithm that better reflects its performance. The new analysis provides significantly lower numbers. They are more consistent with the simulation results of Lin et al. and ours.

In Section II, we review background and related work. The new analysis of the jump-stay rendezvous algorithm, under the asymmetric model, is developed in Section III. Simulation results are presented in Section IV. We conclude with Section V.

\section{BACKGROUND AND RELATED WORK}

The problem of finding and selecting a common channel, by secondary users, can be approached using either a central controller, a dedicated common control channel or a distributed blind rendezvous technique. A blind rendezvous technique may use channel hopping. Each secondary user hops over a set of channels looking to make rendezvous with a peer. Participating users may have a common channel set, under the symmetric 
model, or a different, but non disjoint channel set, under the asymmetric model.

\section{A. The Jump-Stay Rendezvous Algorithm}

Our work leverages the work of Lin et al. They authored the enhanced jump-stay rendezvous algorithm [1], hereafter called the jump-stay rendezvous algorithm. It works for multiple users with guaranteed rendezvous. We illustrate the principle with two users. Time is divided in slots of equal length. A rendezvous takes place within one time slot. It is assumed that the secondary users are synchronous. Each secondary user implements a cyclic behavior. It consists of four phases of the same length, in time slots. The first three phases are identical. The secondary user hops from channel to channel. All channels are visited. Each hop lasts for the duration of one time slot. During the last phase, the secondary user stays on the same channel for the whole phase duration.

Channel hopping is performed according to a pattern determined by the following procedure. Let $m$ be the number of channels (a positive integer). Channel indices are $0, \ldots, m-1$. Let $p$ be the smallest prime number greater than $m$. For instance, if there are four channels, then $p$ is five. Hopping is performed in steps of $r$ units, with $r \in\{1, \ldots, m\}$ and starting index $i \in\{0, \ldots, p-1\}$. Each phase consists of $p$ time slots. In the first phase, hopping is performed for $p$ time slots. The same thing is done in the second and third phases. During the fourth phase, the secondary user stays on channel $r$ for $p$ time slots. The total length of a cycle, called a round, is therefore $4 p$ time slots. Let us index the time slots with variable $t=0,1,2, \ldots, 4 p-1$. As a function of $p, r, i$ and $t$, a number pattern is generated according to

$$
j=(i+t r) \bmod p .
$$

The sequence of generated pattern numbers is such that any window of length $p$ time slots is a permutation of the numbers $0, \ldots, p-1$. The indices of the corresponding channels are produced as

$$
c=j \bmod m \text {. }
$$

Every channel is visited at least once during any interval of $p$ time slots.

Two example jump-stay rendezvous patterns follow:

$\begin{array}{llllllllllllllllllll}0 & 1 & 2 & 3 & 0 & 0 & 1 & 2 & 3 & 0 & 0 & 1 & 2 & 3 & 0 & 1 & 1 & 1 & 1 & 1 \\ 0 & 2 & 0 & 1 & 3 & 0 & 2 & 0 & 1 & 3 & 0 & 2 & 0 & 1 & 3 & 2 & 2 & 2 & 2 & 2\end{array}$

In both examples, $m$ is equal to four and $p$ is equal to five. Each line represents a cyclic behavior. Each number corresponds to a channel visited during a time slot. Each phase consists of five time slots. The channels of the three hopping phases are listed first. The constant channel of the stay phase follows. In the first example, $r$ is equal to one. It is equal to two in the second example. The start index $(i)$ is zero in both examples.

The initial value of the step increment $r$ is selected at random. The initial value of the start index $i$ is also selected at random. It is incremented to the successor value, modulo $p$, after each round. Given a sequence generated with $r=r_{1}$ and another sequence generated with $r=r_{2}$, with $r_{1} \neq r_{2}$, then any jump pattern window of $p$ time slots of the first sequence has a common channel time slot with an overlapping jump pattern window of $p$ time slots of the second sequence [1]. The performance of the algorithm is evaluated in reference to a metric termed the Time-To-Rendezvous (TTR). From the moment both users are running, it is the number of time slots required to achieve rendezvous. Under the two-user symmetric model, the expected TTR of the jump-stay rendezvous algorithm is lower than or equal to $\frac{3 p}{2}+3[1]$. Under the twouser symmetric model, the maximum TTR of the jump-stay rendezvous algorithm is $4 p$ [1]. ${ }^{1}$ An algorithm with a finite maximum TTR is said to be guaranteed rendezvous.

Lin et al. address the followings cases:

1) Two symmetric users: Both users have a common set of available channels (as in the precedent discussion).

2) Two asymmetric users: The users have different sets of available channels. Rendezvous is possible if the sets are not disjoint. While hopping, if a channel determined by the algorithm happens to be unavailable, then an available channel is selected for the hop.

3) Multiple-users multiple-hops (asymmetric or symmetric): Pair-wise rendezvous is repeatedly applied to make global rendezvous between multiple users.

When two users succeed to rendezvous, they exchange their parameters $\left(r_{1}, i_{1}, t_{1}\right)$ and $\left(r_{2}, i_{2}, t_{2}\right)$. An order on the triples is defined. We have $\left(r_{1}, i_{1}, t_{1}\right)<\left(r_{2}, i_{2}, t_{2}\right)$ if $r_{1}<r_{2}$, or $r_{1}=r_{2}$ and $i_{1}<i_{2}$, or $r_{1}=r_{2}$ and $i_{1}=i_{2}$ and $t_{1}<t_{2}$. The largest parameter triple is adopted by both users. In the sequel, the two users hop the same way. In the multiple-user case, this is done repeatedly, pairwise, until all secondary users have adopted the largest parameter triple. In a companion paper [4], we have improved the analysis of the jump-stay rendezvous algorithm under the symmetric model. Hereafter, we develop an analysis for the asymmetric model.

Corollary 1: Let $g$ be the number of channels common to two users, with $1<g \leq m$. Under the asymmetric model, the jump-stay rendezvous algorithm achieves two-user rendezvous in an expected number of rounds lower than or equal to

$$
R=\frac{g}{m^{2}} \cdot 1+\left(1-\frac{g}{m^{2}}\right) \cdot(p+1-g) .
$$

Proof: The proof is structured into two cases, according to whether or not the two users select an equal step increment $(r)$. Let $r_{1}$ be the step increment of the first user and $r_{2}$ be the one of the second user. When $r_{1}$ is equal to $r_{2}$, their step increment corresponds to a common channel index. The two users have $g$ common channels among the $m$ channels. They select the same channel with probability $\frac{g}{m^{2}}$. According to Theorem 1 in [1], the users make rendezvous in one round (left term of the sum). The two users select different step increments with probability $1-\frac{g}{m^{2}}$. When $r_{1}$ and $r_{2}$ are different, the step increments may or may not correspond to common a channel. According to Theorem 3 in [1], the users make rendezvous in a maximum of $p+1-g$ rounds (right term of the sum).

\section{B. Related Work}

Related work includes the random channel, orthogonalsequence-based and modular clock algorithms of Theis et

\footnotetext{
${ }^{1}$ Note that the original non enhanced jump-stay rendezvous algorithm has a slightly better maximum TTR under the symmetric model, i.e., $3 p$, and a slightly worst expected TTR, i.e., $\frac{5 p}{3}+3$.
} 
al. [5], [6]. The random channel algorithm visits all channels in a random order. For each time slot, a channel is picked among the $m$ channels with uniform probability. Under the symmetric model, the expected TTR is $m$ time slots. Under the asymmetric model, the expected TTR is $\mathrm{m}^{2} / \mathrm{g}$. In both models, rendezvous is not guaranteed.

\begin{tabular}{|c|c|c|c|c|c|c|c|c|c|c|c|c|c|}
\hline 0 & 0 & 1 & 2 & 1 & 0 & 1 & 2 & 2 & 0 & 1 & 2 & & \\
\hline & & 0 & 0 & 1 & 2 & 1 & 0 & 1 & 2 & 2 & 0 & 1 & 2 \\
\hline
\end{tabular}

Fig. 1. Orthogonal-sequence-based channel hopping.

With the orthogonal-sequence-based algorithm, channels are visited according to the same pattern by all nodes. By construction, two hopping users are eventually on the same channel. Rendezvous is guaranteed. Let $s_{0}, s_{1}, \ldots, s_{m-1}$ be a permutation of the $m$ channels, the hopping pattern is

$s_{0}, s_{0}, s_{1}, \ldots, s_{m-1}, s_{1}, s_{0}, s_{1}, \ldots, s_{m-1} \ldots s_{m-1}, s_{0}, s_{1}, \ldots, s_{m-1}$.

Two hopping users are illustrated in Figure 1. In that example, $m$ is three. The nodes make rendezvous in the third time slot, from the start of the second user. Rendezvous is guaranteed within $m(m+1)$ time slots.

The modular clock algorithm is analogous to the jump-stay rendezvous algorithm, but the stay pattern is not performed. Two-node rendezvous is guaranteed when they hop using different step increments, i.e., different values for $r$. Because of the absence of the stay pattern, rendezvous never occurs when they start hopping on different channels with identical step increments. To deal with this case, when a node fails to rendezvous for $2 p$ time slots, it switches to a different step increment.

Shin et al. have proposed the channel rendezvous sequence algorithm [7]. Rendezvous is guaranteed to take place. Blind rendezvous approaches can be classified into two different groups according to whether the secondary users are asynchronous or synchronous. The asynchronous user ring-walk algorithm has been proposed by Lin et al. [8], [9]. Preference is given to channels with low interference to primary users. Rendezvous is not guaranteed to take place. The orthogonal sequence and modular clock algorithms do not require synchronization [5], [6]. Asynchronous user algorithms may also work with synchronous users, a special case. Algorithms designed specially for synchronous users include the jump-stay rendezvous algorithm [1]-[3]. Bahl et al. proposed an approach for WiFi/802.11 networks [10]. Rendezvous is guaranteed to take place under the symmetric model. Krishnamurthy et al. proposed a two-phase algorithm [11]. The first phase is for neighbor discovery. It is conducted on common local channels. In the second phase, a global common channel is determined among the participating users. Bian et al. use a quorum principle [12]. They also address the asynchronous case [13], [14]. Rendezvous is guaranteed. They have a solution for a twochannel case. Yang et al. have proposed an algorithm based on the $k$-shift-invariant concept that guarantees rendezvous [15]. Evaluations of the modular clock and random algorithms have been conducted by Robertson et al. using the GNU radio framework [16].

\section{New Analysis Under the AsymmetriC Model}

We derive a new analysis for the expected TTR of the jump-stay rendezvous algorithm that better reflects its performance, for the two-user asymmetric model. Our analysis improves on the results of Lin et al. [1]. The original algorithm remains the same. As a prelude to the analysis of the jumpstay rendezvous algorithm under the asymmetric model, we consider and study a problem that we call $k$ failures in $n$ trials.

\section{A. The Problem of $k$ failures in $n$ trials}

Let us consider an array of $n$ trials, labelled 1 to $n$. Exactly $k$ of them are faulty. The faults are placed in the array independently according to the uniform probability distribution. Let $X$ be the random variable that counts the number of trials until success. Clearly, $X$ can only assume the values 1 to $k+1$. At least one trial is required. A maximum of $k$ consecutive failures are possible before a success. We compute $\operatorname{Pr}[X=i]$, for $i=1, \ldots, k+1$.

Lemma 1: The probability mass function of random variable $X$, i.e., the probability of success on the $i$-th trial, is given by the following equation

$$
\operatorname{Pr}[X=i]=\frac{\left(\begin{array}{c}
k \\
i-1
\end{array}\right)}{\left(\begin{array}{c}
n \\
i
\end{array}\right)} \cdot \frac{n-k}{i} .
$$

Proof: Let $i$ be any integer lower than or equal to $k$. Observe that the first $i$ trials $1,2, \ldots, i$ are faulty if and only if there are exactly $k-i$ faults among the trials $i+1, i+2, \ldots, n$. It follows that

$$
\operatorname{Pr}[X>i]=\operatorname{Pr}[\text { trials } 1 . . i \text { are faulty }]=\frac{\left(\begin{array}{l}
n-i \\
k-i
\end{array}\right)}{\left(\begin{array}{l}
n \\
k
\end{array}\right)} .
$$

It follows that

$$
\begin{aligned}
\operatorname{Pr}[X=i] & =\operatorname{Pr}[X>i-1]-\operatorname{Pr}[X>i] \\
& =\frac{\left(\begin{array}{l}
n-i+1 \\
k-i+1
\end{array}\right)}{\left(\begin{array}{l}
n \\
k
\end{array}\right)}-\frac{\left(\begin{array}{l}
n-i \\
k-i
\end{array}\right)}{\left(\begin{array}{l}
n \\
k
\end{array}\right)} \\
& =\frac{\left(\begin{array}{l}
n-i \\
k-i
\end{array}\right)}{\left(\begin{array}{l}
n \\
k
\end{array}\right)} \cdot \frac{n-k}{k-i+1} \\
& =\frac{\left(\begin{array}{c}
k \\
i-1
\end{array}\right)}{\left(\begin{array}{l}
n \\
k
\end{array}\right)} \cdot \frac{n-k}{i} .
\end{aligned}
$$

This proves the lemma.

In the following theorem, we compute the expected number of trials until the trial at which the first success occurs.

Theorem 1: The expected value of random variable $X$, i.e., the expected number of trials until the trial at which the first success occurs, is given by the formula

$$
E[X]=\frac{n+1}{n+1-k} .
$$


Proof: Using Identity (1), we conclude that

$$
\begin{aligned}
E[X] & =\sum_{i=1}^{k+1} i \cdot \operatorname{Pr}[X=i] \\
& =(n-k) \sum_{i=1}^{k+1} \frac{\left(\begin{array}{c}
k \\
i-1
\end{array}\right)}{\left(\begin{array}{c}
n \\
i
\end{array}\right)} .
\end{aligned}
$$

Next we use the Identity (5) and a technique described by Graham et al. [17] [Problem 1, page 173] for computing a sum of quotients of binomial coefficients.

First of all observe that in Identity (5) we can reduce the dependency on $i$ in the denominator of the fraction occurring in the summand by using a simple transformation of the binomial coefficients. Namely, Identity (5) for the expectation can be transformed as follows.

$$
\begin{aligned}
E[X] & =(n-k) \sum_{i=1}^{k+1} \frac{\left(\begin{array}{c}
k \\
i-1
\end{array}\right)}{\left(\begin{array}{c}
n \\
i
\end{array}\right)} \\
& =(n-k) \sum_{i=1}^{k+1} \frac{\left(\begin{array}{l}
k \\
i
\end{array}\right)}{\left(\begin{array}{c}
n \\
i
\end{array}\right)} \cdot \frac{i}{k-i+1} \\
& =(n-k) \sum_{i=1}^{k+1} \frac{\left(\begin{array}{l}
n-i \\
k-i
\end{array}\right)}{\left(\begin{array}{c}
n \\
k
\end{array}\right)} \cdot \frac{i}{k-i+1} \\
& =\frac{n-k}{\left(\begin{array}{l}
n \\
k
\end{array}\right)} \sum_{i=1}^{k+1}\left(\begin{array}{l}
n-i \\
k-i
\end{array}\right) \cdot \frac{i}{k-i+1} \\
& =\frac{1}{\left(\begin{array}{l}
n \\
k
\end{array}\right)} \sum_{i=1}^{k+1}\left(\begin{array}{c}
n-i \\
k+1-i
\end{array}\right) \cdot i .
\end{aligned}
$$

Observe that Identity (6) is sufficient to conclude the proof of the theorem, if we could prove the following claim.

a) Claim.: The identity

$$
\sum_{i=1}^{k+1}\left(\begin{array}{c}
n-i \\
k+1-i
\end{array}\right) \cdot i=\left(\begin{array}{c}
n+1 \\
k
\end{array}\right)
$$

is valid.

b) Proof of Identity (7).: First of all, observe the validity of the identity

$$
\left(\begin{array}{c}
n-i \\
k+1-i
\end{array}\right) \cdot(n+1-i)=\left(\begin{array}{c}
n+1-i \\
n-k
\end{array}\right) \cdot(n-k),
$$

which after multiplying out and collecting terms can be used to derive the identity

$$
\left(\begin{array}{c}
n-i \\
k+1-i
\end{array}\right) \cdot i=\left(\begin{array}{c}
n-i \\
k+1-i
\end{array}\right) \cdot(n+1)-\left(\begin{array}{c}
n+1-i \\
n-k
\end{array}\right) \cdot(n-k) .
$$

If we replace the summand in Identity (7) with the formula derived in Identity (9) we see that

$$
\begin{aligned}
& \sum_{i=1}^{k+1}\left(\begin{array}{c}
n-i \\
k+1-i
\end{array}\right) \cdot i= \\
& \quad(n+1) \cdot \sum_{i=1}^{k+1}\left(\begin{array}{c}
n-i \\
k+1-i
\end{array}\right)-(n-k) \cdot \sum_{i=1}^{k+1}\left(\begin{array}{c}
n+1-i \\
n-k
\end{array}\right) .
\end{aligned}
$$

Next we use the well-known identity

$$
\sum_{k \leq m}\left(\begin{array}{c}
n-m+k \\
k
\end{array}\right)=\left(\begin{array}{c}
n+1 \\
m
\end{array}\right),
$$

which can be found in [17] [page 173] so as to replace the two sums of binomial coefficients in the righthand side of Identity (10) with simpler binomial coefficients. Indeed, using elementary calculations we derive the following identity

$\sum_{i=1}^{k+1}\left(\begin{array}{c}n-i \\ k+1-i\end{array}\right) \cdot i=(n+1) \cdot\left(\begin{array}{l}n+1 \\ k+1\end{array}\right)-(n-k) \cdot\left(\begin{array}{l}n+2 \\ k+1\end{array}\right)$.

Finally, the righthand side of Identity (12) is trivially shown to satisfy

$$
(n+1) \cdot\left(\begin{array}{l}
n+1 \\
k+1
\end{array}\right)-(n-k) \cdot\left(\begin{array}{c}
n+2 \\
k+1
\end{array}\right)=\left(\begin{array}{c}
n+1 \\
k
\end{array}\right),
$$

which completes the proof of the claim made in Identity (7). Because,

$$
\frac{\left(\begin{array}{c}
n+1 \\
k
\end{array}\right)}{\left(\begin{array}{c}
n \\
k
\end{array}\right)}=\frac{n+1}{n+1-k}
$$

the proof of the theorem is complete.

We investigate the concentration of the random variable $X$. Using Identity (2), we see that

$$
\begin{aligned}
\operatorname{Pr}[X>i] & =\frac{\left(\begin{array}{l}
n-i \\
k-i
\end{array}\right)}{\left(\begin{array}{l}
n \\
k
\end{array}\right)} \\
& =\frac{k(k-1) \cdots(k-i+1)}{n(n-1) \cdots(n-i+1)} \\
& \leq\left(\frac{k-i+1}{n-i+1}\right)^{i},
\end{aligned}
$$

where the last inequality holds since $\frac{k-j+1}{n-j+1}$ is monotone increasing in $j$. Also, in view of Identity (4), we have

$$
E[X]=\frac{n+1}{n+1-k}=\frac{1}{1-\frac{k}{n+1}} .
$$

Notice that the previously discussed model resembles the model of independent Bernoulli trials. Assume that a trial fails randomly and independently with probability $q$, for some $0<$ $q<1$. Let $X$ be a random variable that counts the number of trials until the trial at which the first success occurs. It is well-known that

$$
\begin{aligned}
& \operatorname{Pr}[X>t]=q^{t} \\
& \operatorname{Pr}[X=t]=q^{t-1}(1-q) .
\end{aligned}
$$

Moreover,

$$
E[X]=\frac{n q^{n+1}-(n+1) q^{n}+1}{1-q}=\frac{1-q^{n}}{1-q}-n q^{n},
$$

and therefore

$$
E[X] \rightarrow \frac{1}{1-q}, \text { as } n \rightarrow \infty .
$$

From this, one can see the similarity of Identities (13) and (14) with Identities (15) and (17), respectively. 


\section{B. Expected Number of Rounds Under the Asymmetric Model}

Corollary 2: Let $g$ be the number of channels common to two users, with $1<g \leq m$. Under the two-user asymmetric model, the expected number of rounds $R$ required to make two-user rendezvous with the jump-stay rendezvous algorithm is

$$
R=\frac{g}{m^{2}} \cdot 1+\left(1-\frac{g}{m^{2}}\right) \cdot \frac{p+1}{1+g} .
$$

Proof: If $r_{1}$ and $r_{2}$ are equal, then one round is required (Theorem 3 in [1]). This case probability is $\frac{g}{m^{2}}$. If $r_{1}$ and $r_{2}$ are different, then there is a possibility of $p-g$ failures in $p$ trials, or rounds. This case probability is $1-\frac{g}{m^{2}}$. This can be seen as an instance of the problem of $k$ failures among $n$ trials (Subsection III-A), placed independently according to the uniform probability distribution, where $n$ is equal to $p$ and $k$ is equal to $p-g$. According to Theorem 1, the expected value of $p$ trials with $p-g$ failures is

$$
\frac{n+1}{n+1-k}=\frac{p+1}{p+1-p+g}=\frac{p+1}{1+g} .
$$

Theorem 2: Under the two-user asymmetric model, the expected TTR of the jump-stay rendezvous algorithm is lower than or equal to $R \cdot 4 p$, with $R$ as defined in Corollary 2 .

Proof: It follows from Corollary 2 and the fact that a round is $4 p$ time slots.

For the two-user asymmetric case, note that the expected TTR derived in Corollary 2 is lower than or equal to the one derived in Lin et al. [1], i.e., $4 p(p+1-g)-[4 p g(p-g)+g / 2] /\left(m^{2}\right)$, see the plots in the next section.

\section{Simulations}

Simulations were conducted using the $\mathrm{OMNeT}++$ framework [18]. Under the two-user symmetric model, we evaluate the performance of the jump-stay rendezvous algorithm. Results are compared with the analysis of Section III. Each simulation consists of 10 to 100 -channel scenarios. Two-user and multiple-user rendezvous scenarios are included. The TTRs are measured using 500 runs and $95 \%$ confidence intervals.

Figure 2 plots the expected TTR according to the equation of Corollary 1 for two-user rendezvous in 10 to 100-channel scenarios. $10 \%$ to $90 \%$ of the available channels are common to both users. Figure 3 plots the expected TTR under the same conditions, but according to the equation of Theorem 2.

Figure 4 depicts the jump-stay rendezvous algorithm simulation results, under the asymmetric model for two, 10 and 20-user scenarios. Comparing with Figure 2, we can see that the expected TTR of Lin et al. for the asymmetric case is highly over estimated. The $95 \%$ confidence intervals of the OMNeT++ simulation results, especially in the two-user scenarios, show the low stability of the jump-stay rendezvous algorithm in the asymmetric case when the number of common channels decreases.

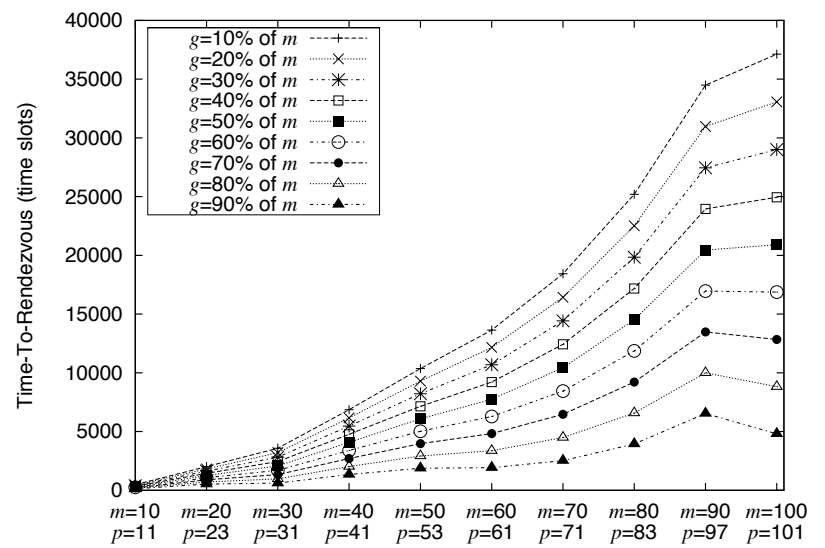

Fig. 2. Expected TTR for asymmetric two-user rendezvous in 10 to 100channel scenarios according to Corollary 1 .

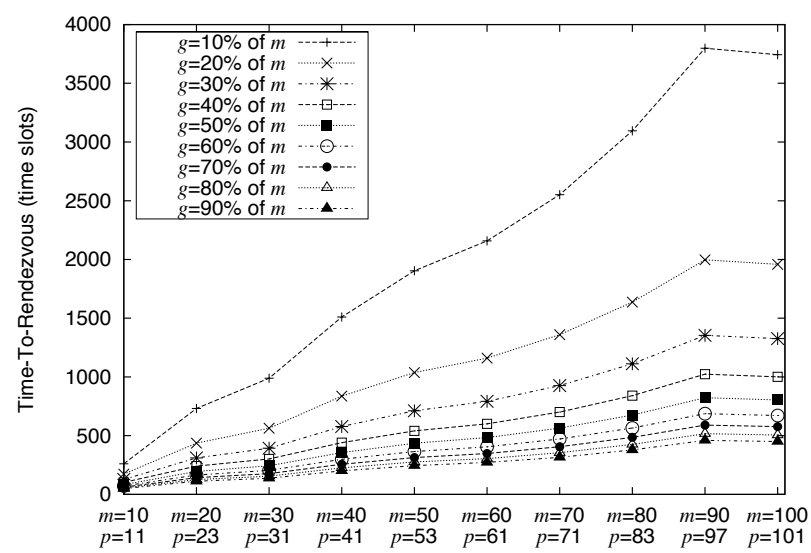

Fig. 3. Expected TTR for asymmetric two-user rendezvous in 10 to 100channel scenarios according to the analytic model of Theorem 2 .

\section{CONCLUSION}

We developed a new analysis for the expected TTR of the jump-stay rendezvous algorithm of Lin et al. [1], under the asymmetric two-user model. Their original algorithm is unchanged. The new analysis is more consistent with the simulation results, the ones of Lin et al. and ours.

\section{ACKNOWLEDGMENT}

We acknowledge financial support from Natural Sciences and Engineering Research Council of Canada, Spanish Ministry of Science (projects CONSOLIDER INGENIO 2010 CSD2007-0004 ARES and TIN2011-27076-C03-02 COPRIVACY) and Innovation and Ministry of Education of Mexico. The authors thank A. Dotor for all his help on the simulations reported in Section IV. 


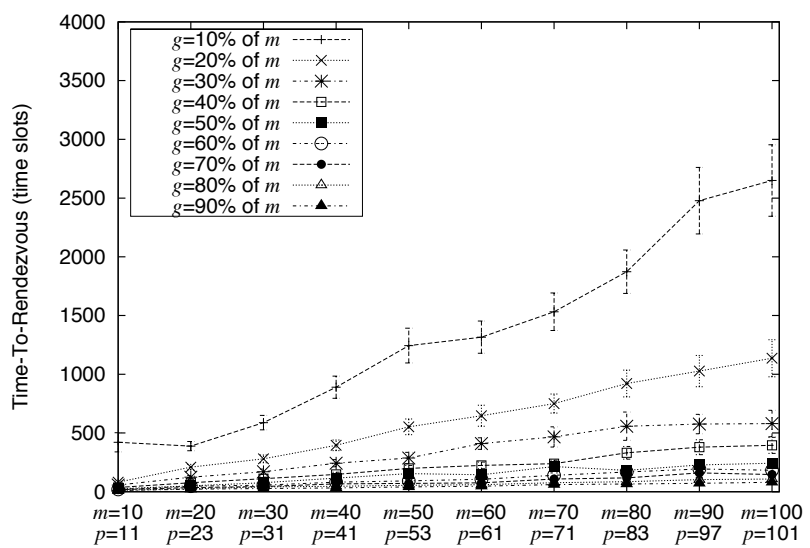

(a) Two users.

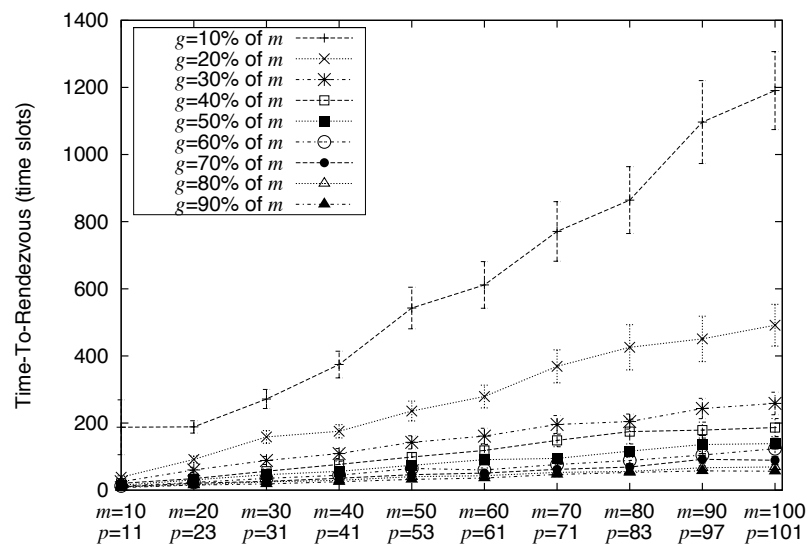

(b) 10 users

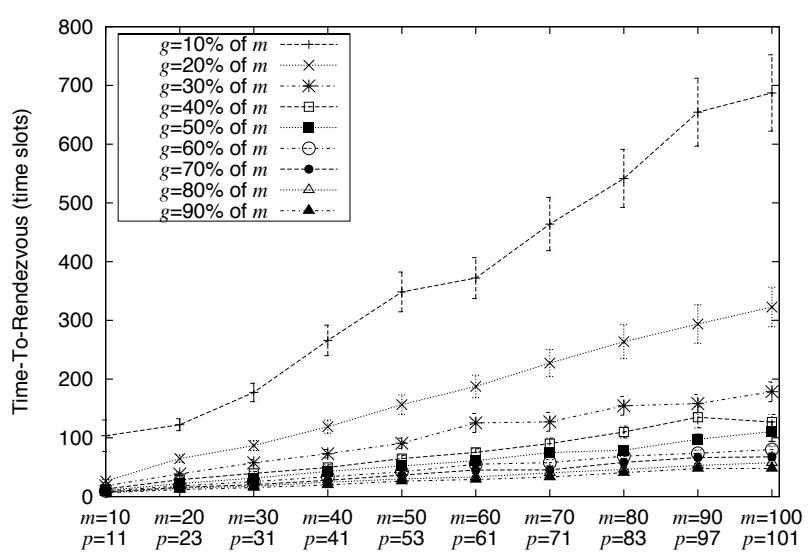

(c) 20 users

Fig. 4. Average TTR for asymmetric two, 10 and 20 users executing the jump-stay rendezvous algorithm in 10 to 100 -channel scenarios obtained with the OMNeT++ simulations.

\section{REFERENCES}

[1] Z. Lin, H. Liu, X. Chu, and Y. Leung, "Enhanced jump-stay rendezvous algorithm for cognitive radio networks," IEEE Communications Letters, pp. 1-4, 2013.

[2] Z. Lin, H. Liu, X. Chu, and Y.-W. Leung, "Jump-stay based channelhopping algorithm with guaranteed rendezvous for cognitive radio networks," in Proceedings of IEEE INFOCOM, April 2011, pp. 24442452.

[3] H. Liu, Z. Lin, X. Chu, and Y.-W. Leung, "Jump-stay rendezvous algorithm for cognitive radio networks," IEEE Transactions on Parallel and Distributed Systems, vol. 23, no. 10, pp. 1867-1881, 2012.

[4] M. Barbeau, G. Cervera, J. Garcia-Alfaro, and E. Kranakis, "A new analytic model for the cognitive radio jump-stay algorithm," in Wireless Days, 2013.

[5] N. Theis, R. Thomas, and L. DaSilva, "Rendezvous for cognitive radios," Mobile Computing, IEEE Transactions on, vol. 10, no. 2, pp. 216-227, 2011.

[6] L. DaSilva and I. Guerreiro, "Sequence-based rendezvous for dynamic spectrum access," in 3rd IEEE Symposium onNew Frontiers in Dynamic Spectrum Access Networks (DySPAN), 2008, pp. 1-7.

[7] J. Shin, D. Yang, and C. Kim, "A channel rendezvous scheme for cognitive radio networks," IEEE Communications Letters, vol. 14, no. 10, pp. 954-956, 2010.

[8] Z. Lin, H. Liu, X. Chu, and Y. . Leung, "Ring-walk rendezvous algorithms for cognitive radio networks," Ad-Hoc and Sensor Wireless Networks, vol. 16, no. 4, pp. 243-271, 2012.

[9] H. Liu, Z. Lin, X. Chu, and Y.-W. Leung, "Ring-walk based channelhopping algorithms with guaranteed rendezvous for cognitive radio networks," in IEEE/ACM Int'l Conference on Cyber, Physical and Social Computing (CPSCom), Green Computing and Communications (GreenCom), 2010, pp. 755-760.

[10] P. Bahl, R. Chandra, and J. Dunagan, "SSCH: slotted seeded channel hopping for capacity improvement in IEEE 802.11 ad-hoc wireless networks," in Proceedings of the 10th annual international conference on Mobile computing and networking, ser. MobiCom. New York, NY, USA: ACM, 2004, pp. 216-230. [Online]. Available: http://doi.acm.org/10.1145/1023720.1023742

[11] S. Krishnamurthy, M. Thoppian, S. Kuppa, R. Chandrasekaran, N. Mittal, S. Venkatesan, and R. Prakash, "Time-efficient distributed layer-2 auto-configuration for cognitive radio networks," Computer Networks, vol. 52, no. 4, pp. 831-849, 2008.

[12] K. Bian, J.-M. Park, and R. Chen, "A quorum-based framework for establishing control channels in dynamic spectrum access networks," in Proceedings of the 15th annual international conference on Mobile computing and networking, ser. MobiCom. New York, NY, USA: ACM, 2009, pp. 25-36. [Online]. Available: http://doi.acm.org/10.1145/1614320.1614324

[13] K. Bian and J.-M. Park, "Asynchronous channel hopping for establishing rendezvous in cognitive radio networks," in Proceedings of IEEE INFOCOM, 2011, pp. 236-240.

[14] _ "Maximizing rendezvous diversity in rendezvous protocols for decentralized cognitive radio networks," IEEE Transactions on Mobile Computing, vol. 12, no. 7, pp. 1294-1307, 2013.

[15] D. Yang, J. Shin, and C. Kim, "Deterministic rendezvous scheme in multichannel access networks," Electronics Letters, vol. 46, no. 20, pp. 1402-1404, 2010.

[16] A. Robertson, L. Tran, J. Molnar, and E.-H. Fu, "Experimental comparison of blind rendezvous algorithms for tactical networks," in World of Wireless, Mobile and Multimedia Networks (WoWMoM), 2012 IEEE International Symposium on a, 2012, pp. 1-6.

[17] R. L. Graham, D. E. Knuth, and O. Patashnik, Concrete mathematics a foundation for computer science. Addison-Wesley Reading, 1989.

[18] A. Varga and R. Hornig, "An overview of the OMNeT++ simulation environment," in 1st International conference on Simulation tools and techniques for communications, networks and systems \& workshops (Simutools), 2008. 